\title{
Preoperative Imaging for Thoracic Branch of Supraclavicular Artery Flap
}

\author{
A Comparative Study of Contrast-Enhanced Ultrasound With Three-Dimensional \\ Reconstruction and Color Duplex Ultrasound
}

\author{
Yashan Gao, MD, * Yuwen Yuan, MD, * Haizhou Li, MD, * Bin Gu, MD, * Feng Xie, MD, * Tanja Herrler, MD, † \\ Qingfeng Li, MD, PhD, * and Tao Zan, MD, PhD*
}

\begin{abstract}
Objective: The thoracic branch of supraclavicular artery (TBSA) flap has been widely used to reconstruct face and neck defects. However, the branches of the supraclavicular artery (SCA) exhibit considerable anatomical variations. The aim of this study was to evaluate and compare the role of contrast-enhanced ultrasound (CEUS) with 3-dimensional (3D) reconstruction and regular color duplex ultrasonography (CDUS) in the preoperative assessment of TBSA flap.

Methods: From May 2009 to October 2013, 20 patients (involving 26 flaps) receiving anterior chest flaps for lower face and neck reconstruction underwent both CDUS and CEUS with 3D reconstruction preoperatively for detecting the TBSAs. The number of TBSAs, their caliber, peak systolic velocity (PSV), and course were recorded. In case of an absent TBSA, the second and third perforators of the internal mammary artery were detected. The preoperative imaging data were compared with the intraoperative findings to evaluate the value of CDUS and CEUS with 3D reconstruction for planning and performing the TBSA flaps. All patients were followed up for more than 1 year.

Results: A total of 37 TBSAs in 16 flaps were found by CDUS with a mean caliber of $0.6 \pm 0.1 \mathrm{~mm}$ and a mean PSV of $13.1 \pm 1.6 \mathrm{~cm} / \mathrm{s}$, whereas 48 TBSAs in 20 flaps were found by CEUS with a mean caliber of $0.8 \pm 0.2 \mathrm{~mm}$ and a mean PSV of $12.5 \pm 2.1 \mathrm{~cm} / \mathrm{sec}$. In 18 flaps with TBSA PSV above $10 \mathrm{~cm} / \mathrm{s}$, pedicled TBSA flaps were performed, whereas pedicled or free internal mammary artery flaps were chosen as alternative for the remaining 8 flaps. All 48 TBSAs were found intraoperatively and their origin from the SCA confirmed, indicating specificity and positive predictive value of both CDUS and CEUS were $100 \%$ in localizing TBSA preoperatively, whereas sensitivity and negative predictive value of CEUS were higher than using CDUS.

Conclusions: The branches of SCA have marked anatomical variations. CEUS with $3 \mathrm{D}$ reconstruction has advantages over CDUS for the preoperative assessment of the donor-site vascular supply of TBSA flaps.
\end{abstract}

Key Words: contrast-enhanced ultrasound, color duplex ultrasonography, thoracic branch of supraclavicular artery flap, preexpanded anterior chest flap

(Ann Plast Surg 2016;77: 201-205)

$\mathrm{P}$ ostburn scar in the face and neck region significantly impact appearance and function of the face and may further cause severe psychosocial problems. Based on the special anatomic characteristics in

Received March 4, 2015, and accepted for publication, after revision June 18, 2015. From the *Department of Plastic and Reconstructive Surgery, Shanghai Ninth People's Hospital, Shanghai Jiao Tong University School of Medicine, Shanghai, P. R. China; and †Hand, Plastic, Aesthetic Surgery, Department of General, Trauma, Hand, Plastic Surgery, University of Munich, Munich, Germany.

T.Z. and Q.L. equally contributed to this work and should be considered as cocorresponding authors.

Conflicts of interest and sources of funding: This work was supported by grants from the National Natural Science Foundation of China $(81471857,81101437)$, the National Key Project of Scientific and Technical Supporting Programs Funded by the Ministry of Science \& Technology of China (2012BAI11B03).

Reprints: Tao Zan, MD, PhD and Qingfeng Li, MD, PhD, Department of Plastic and Reconstructive Surgery, Shanghai Ninth People's Hospital, Shanghai Jiao Tong University School of Medicine, 639 Zhizaoju Rd, Shanghai 200011, P.R. China. E-mail: zantaodoctor@yahoo.com; dr.liqingfeng@yahoo.com.

Copyright (C) 2015 Wolters Kluwer Health, Inc. All rights reserved.

ISSN: 0148-7043/16/7702-0201

DOI: $10.1097 /$ SAP.0000000000000601 this area, we have proposed the principle of "matching, large size, and thinner thickness" in flap selection for facial reconstruction. ${ }^{1}$ According to this principle, preexpanded anterior chest flaps appear to be the ideal choices for cervicofacial reconstruction. Different types of anterior chest flaps can be used, among which the thoracic branch of supraclavicular artery (TBSA) flap is a workhorse flap. ${ }^{2}$

The supraclavicular artery (SCA) originates from the transverse cervical artery in a triangle formed by the external jugular vein, sternocleidomastoid muscle and clavicle, enters the subcutaneous layer of the supraclavicular region, and divides laterally and caudally into deltoid branch and thoracic branches. The deltoid branch extends to the acromial region and could be harvested as "SCA island flap (SCAIF)", whereas the thoracic branches traverse the clavicular to the thoracic region and may be elevated as TBSA flaps. ${ }^{2-4}$ However, Ma et $\mathrm{al}^{5}$ identified 4 types of SCA and found that the thoracic branch only existed in $60 \%$ of the cases. Therefore, preoperative assessment of the vessels is essential for successful flap transfer. However, currently used preoperative mapping methods, such as handheld Doppler (HHD), color duplex ultrasonography (CDUS), computed tomography angiography (CTA), and magnetic resonance angiography (MRA), have shown considerable drawbacks. ${ }^{6-12}$

In this study, we propose a new method for preoperative assessment of perforators using contrast-enhanced ultrasound (CEUS) with $3 \mathrm{D}$ reconstruction, which is minimally invasive, nonexpensive and can accurately detect vessels with a diameter less than $1.0 \mathrm{~mm}$, differentiate the arteries and veins, and provide the 3D images. We compared the accuracy of CEUS with 3D reconstruction and traditional CDUS in detecting TBSA and discussed the variation of SCA using CEUS with $3 \mathrm{D}$ reconstruction.

\section{PATIENTS AND METHODS}

\section{Patients}

From May 2009 to October 2013, 20 patients (26 flaps) between 20 and 45 years of age underwent surgery to resurface postburn neck, cheek, or perioral defects using preexpanded anterior chest flaps. Preoperatively, pedicle vessels were evaluated using both CDUS and CEUS with 3D reconstruction. The protocol of this study was approved by the local ethics committee, and all patients gave informed consent. All procedures were performed at our institution by the same surgeons and ultrasound team. All patients were followed up for more than 1 year.

\section{Tissue Expansion}

All patients received tissue expander implantation in the first stage to achieve a larger and thinner flap and to reduce donor-site morbidity. An incision was made lateral to the expander pocket and anterior to the deltopectoral groove. The dissection was performed between the subcutaneous tissue and the pectoralis fascia. A rectangular tissue expander was then implanted into the anterior chest pocket. The sequential water instillation procedure was performed twice a week for 
TABLE 1. Number, Caliber, and PSV of TBSA in 26 Flaps Detecting by CDUS and CEUS

\begin{tabular}{|c|c|c|c|c|}
\hline & \multicolumn{2}{|c|}{ Number (Branch) } & \multirow{2}{*}{$\begin{array}{c}\text { Caliber, mm } \\
0.6 \pm 0.1\end{array}$} & \multirow{2}{*}{$\frac{\text { PSV, } \mathbf{~ c m} / \mathbf{s}}{13.1 \pm 1.6}$} \\
\hline CDUS & & 37 & & \\
\hline \multirow[t]{3}{*}{ CEUS } & $48^{*}$ & 37 & $0.9 \pm 0.1$ & $13.4 \pm 1.5$ \\
\hline & & $P$ & $<0.05 \dagger$ & $>0.05 \ddagger$ \\
\hline & & 11 & $0.6 \pm 0.1$ & $9.3 \pm 1.1$ \\
\hline
\end{tabular}

*48 TBSAs detected by CEUS included 37 branches also found by CDUS and further 11 branches found by CEUS.

† Significant differences exited between CDUS and CEUS measures of the caliber of 37 branches.

\$No significant differences exited between CDUS and CEUS measures of the PSV of 37 branches.

4 to 8 months starting 2 weeks postoperatively until the estimated expanded skin size exceeded the size of the tissue defect.

\section{Ultrasound Examination}

A GE Voluson E8 ultrasonic device (GE Health-care, Little Chalfont, United Kingdom) with a 10- to $18-\mathrm{MHz}$ liner probe and a volume probe was used in the present investigation. A 7.5-MHz color Doppler flowmeter with its color Doppler flow imaging (CDFI) set in the most sensitive and least noise-generating mode was used for both CDUS and CEUS.

\section{Vessel Evaluation}

The deltoid and thoracic branches of the SCA were detected in the supraclavicular and subclavicular region by both CDUS and CEUS with $3 \mathrm{D}$ reconstruction according to their anatomical characteristics with the patient in a supine position. In case of an absent TBSA, the second and third internal mammary arteries (IMAP), which penetrate the muscular layer in the intercostal spaces $1 \mathrm{~cm}$ lateral to the sternum and proceed toward the deltopectoral groove, were detected. The location and course of these vessels were identified and marked on the body surface. Vessel quality was measured by peak systolic velocity (PSV) and caliber.

\section{CEUS and 3D Reconstruction}

The contrast agent SonoVue (Bracco, Milan, Italy) was dissolved in $10 \mathrm{~mL}$ of saline and injected through ulnar vein at a speed of $1 \mathrm{~mL} / \mathrm{min}$. It was adopted to gain the best outcome with a significant signal increase but with the least CDFI signal excess. The contrast agent SonoVue based on sulfur hexafluoride-microbubbles could raise the blood echo and enhances the signal-to-noise ratio to improve the vessel image. After injection of the contrast agent, the dotted signals of CDUS were converted into a continual line, and the vessels were more visible. $^{13,14}$ We then performed $3 \mathrm{D}$ ultrasound scanning and $3 \mathrm{D}$ reconstruction of the vascular network using the volume probe which clearly showed the course of the vessels and their surrounding anatomy.

\section{Intraoperative Evaluation of Perforators}

According to our proposed treatment algorithm for face and neck reconstruction using anterior chest flaps, ${ }^{2}$ the pedicled TBSA flap was preferred for resurfacing lower face and neck defect because of its closer pivot point and long pedicle length. According to our clinical experience, the TBSA of which the PSV was above $10 \mathrm{~cm} / \mathrm{sec}$ could be selected as the pedicle. In case of an absent TBSA, the IMAP flap was used as an alternative option, which is more reliable. In this study, TBSA and IMAP flaps were harvested after confirmation of the perforators by ultrasound examination. The courses of the perforators were checked intraoperatively and compared with the CDUS and CEUS images to evaluate the accuracy of CDUS and CEUS in detecting perforators in the anterior chest region. The distance between the preoperative outcomes and intraoperative findings was measured in millimeters.

\section{Statistical Analysis}

TBSA caliber and PSV showed normal distributions. Measures of these variables were reported as means \pm standard deviations. The student $t$ test was adopted to assess significant differences. A P value less than 0.05 was considered to indicate a significant difference. Statistical analyses were performed with SPSS 19.0.

\section{RESULTS}

A total of 37 TBSAs in 16 flaps were found by CDUS with a mean caliber of $0.6 \pm 0.1 \mathrm{~mm}$ and a mean PSV of $13.1 \pm 1.6 \mathrm{~cm} / \mathrm{sec}$. After injection of the contrast agent SonoVue, the CDFI blood flow signal of all these 37 branches were enhanced with a mean caliber of $0.9 \pm$ $0.1 \mathrm{~mm}$ and a mean PSV of $13.4 \pm 1.5 \mathrm{~cm} / \mathrm{sec}$. In addition, we further identified another 11 TBSAs with a mean caliber of $0.6 \pm 0.1 \mathrm{~mm}$ and a mean PSV of $9.3 \pm 1.1 \mathrm{~cm} / \mathrm{sec}$. In summary, 48 TBSAs in 20 flaps were found by CEUS with a mean caliber of $0.8 \pm 0.2 \mathrm{~mm}$ and a mean PSV of $12.5 \pm 2.1 \mathrm{~cm} / \mathrm{sec}$ (Table 1). Also, using the volume probe and the $3 \mathrm{D}$ reconstruction method, we were able to obtain continuous stereoscopic vascular imaging which provided a clearer course of the vessels, as well as their branching and distribution in the soft tissue.

Detected vessels were classified into SCA type I to IV as previously described by Ma et al. ${ }^{5}$ Briefly, type I included a pronounced deltoid branch, whereas the TBSA was thin or absent. In type II, the deltoid and thoracic branches were equal. In type III, the thoracic branch represented the main branch. Type IV was characterized by a thoracic branch originating from the transverse cervical artery. Among the 26 flaps investigated by CEUS in this study, 8 flaps were classified in type I with a TBSA PSV less than $10 \mathrm{~cm} / \mathrm{s}$, type II was found in 10 cases, 7 cases were attributed to type III, and 1 case to type IV (Fig. 1). After all, the TBSAs with PSV above $10 \mathrm{~cm} / \mathrm{sec}$ were found in 18 of the
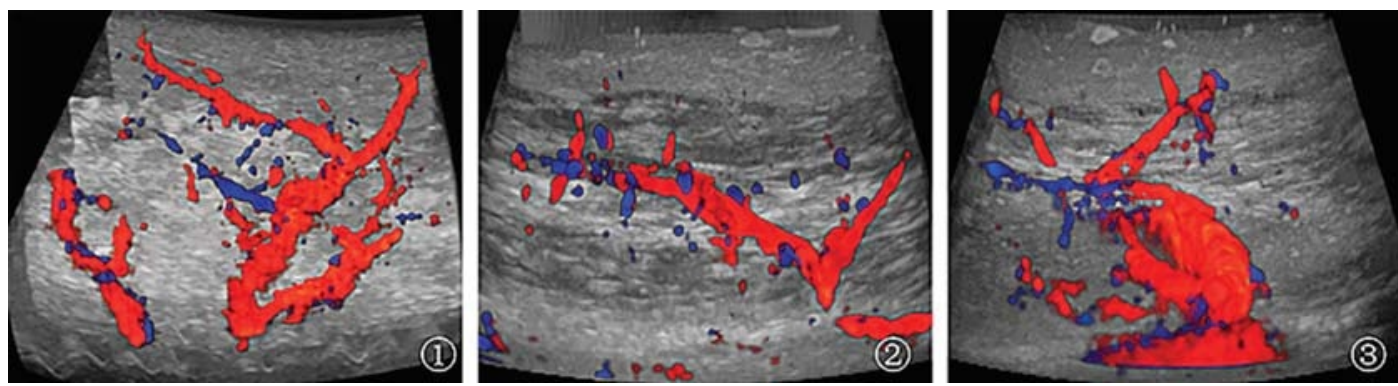

FIGURE 1. Contrast-enhanced ultrasound with 3D reconstruction showed the anatomical variations of supraclavicular artery. (1) The deltoid branch was as big as the thoracic branch. (2) The deltoid branch was big and main. (3) The thoracic branch was big and main. 
TABLE 2. Performance of CDUS and CEUS With 3D Reconstruction in Predicting the Presence of TBSAs

\begin{tabular}{|c|c|c|c|c|c|c|c|}
\hline \multirow[b]{2}{*}{ US signal } & \multicolumn{3}{|c|}{ Flaps With TBSAs } & \multirow[b]{2}{*}{ Sensitivity (\%) } & \multirow[b]{2}{*}{ Specificity (\%) } & \multirow[b]{2}{*}{ Positive Predictive Value (\%) } & \multirow[b]{2}{*}{ Negative Predictive Value (\%) } \\
\hline & + & - & Total & & & & \\
\hline \multicolumn{8}{|l|}{ CDUS } \\
\hline+ & 16 & 0 & 16 & 80 & 100 & 100 & 60 \\
\hline- & 4 & 6 & 10 & & & & \\
\hline Total & 20 & 6 & 26 & & & & \\
\hline \multicolumn{8}{|l|}{ CEUS } \\
\hline+ & 20 & 0 & 20 & 100 & 100 & 100 & 100 \\
\hline- & 0 & 6 & 6 & & & & \\
\hline Total & 20 & 6 & 26 & & & & \\
\hline
\end{tabular}

26 flaps, and we could design the pedicled TBSAs flaps based on the CEUS information.

All 26 flaps in this study were preexpanded anterior chest flaps and performed to resurface postburn neck, cheek, and/ or perioral defects. For the 18 flaps in which TBSAs were preoperatively found to exhibit above $10 \mathrm{~cm} / \mathrm{s}$, pedicled TBSA flaps were designed. For the other 8 flaps, pedicled or free IMAP flaps were chosen as alternative after confirmation of IMAP by ultrasound examination. ${ }^{2}$ Intraoperatively, the TBSAs of all 26 flaps were checked to evaluate and compare the accuracy of CDUS and CEUS in detecting TBSA. All 37 TBSAs detected by CDUS and 48 TBSAs detected by CEUS were found intraoperatively, and their origin from SCA confirmed. The distance between the preoperatively marked location and the actual intraoperative position was maximum $0.8 \mathrm{~mm}$. No further TBSA was found intraoperatively. These results indicated the specificity and positive predictive value of both CDUS and CEUS were $100 \%$ in localizing TBSA preoperatively, whereas the sensitivity and negative predictive value of CEUS were higher than that of CDUS (Table 2). All flaps were harvested successfully according to our preoperative planning except for 1 case in which the TBSA detected by CEUS with a caliber of $0.6 \mathrm{~mm}$ was intraoperatively found too small and therefore switched to a free IMAP flap for reconstruction, suggesting the measurement error of CEUS to evaluate vessel caliber, which may be caused by the CDFI signal excess.

Complications related to the contrast agent included minor gastrointestinal discomfort in 1 patient who recovered within 1 day after without special treatment. The donor sites were closed primarily in all cases without complication. Flap transfer complications included tip congestion and necrosis in 2 cases. All the patients were followed up for more than 1 year without further complications and were satisfied with the surgical outcomes.

\section{Case Report}

A 29-year-old man presented with postburn scar contractures of neck and lower face. Bilateral preexpanded pedicled TBSA flaps were indicated to resurface the defect of neck and lower face beginning with the left side. The CDUS examination revealed one left TBSA running in the subdermal layer of the expanded flap with a caliber of $0.7 \mathrm{~mm}$ and PSV of $12.1 \mathrm{~cm} / \mathrm{sec}$. After injection of the contrast agent SonoVue, the CDFI signal of the TBSA increased. The CEUS showed the TBSA more clearly with a caliber of $1.0 \mathrm{~mm}$ and PSV of $13 \mathrm{~cm} / \mathrm{sec}$. Using the $3 \mathrm{D}$ reconstruction method, we were able to visualize the complete course of the TBSA and its root vessel, the left SCA. Based on the information from CEUS with 3D reconstruction, we designed the pedicled TBSA flap. During flap harvesting, the location of the TBSA was confirmed as identified preoperatively. No complication related to contrast agent occurred. The donor site was closed primarily, and the flap survived completely (Fig. 2).

\section{DISCUSSION}

Cervicofacial reconstruction with preexpanded anterior chest flaps exhibits several advantages. First, this area provides skin and soft tissue matching face and neck in color, texture, and thickness. Second, using soft-tissue expansion, we were able to harvest a larger and thinner flap and simultaneously reduce donor site morbidity. According to the principle of "matching, large size, and thinner thickness" in flap selection for facial reconstruction, ${ }^{1}$ the preexpanded anterior chest flaps represent an ideal treatment option. Skin and soft tissue of the anterior chest are primarily nourished by the SCA, the internal mammary artery, the thoracoacromial artery, and the lateral thoracic artery. ${ }^{15}$ A pedicled TBSA flap, IMAP flap, and thoracoacromial artery perforator flap ${ }^{16}$ may be harvested in this area. The TBSA flap was firstly described by Ma et $\mathrm{al}^{17}$ in 1994. Later, many surgeons reported the use of TBSA flaps for cervicofacial reconstruction with excellent utcomes. ${ }^{2,4,18}$ The pedicled preexpanded TBSA flap is preferred over other flaps for the reconstruction of the neck and lower face due to the advantageous location of the TBSA pivot point approximately $2 \mathrm{~cm}$ cranially of the clavicular midpoint and, thus, close to the face. ${ }^{2}$ Moreover, sufficient TBSA pedicle length may always obtained by ligation of the terminal branches of the transverse cervical artery. However, a dominant TBSA does not exist in every patient. According to the cadaver study by Ma et al, the TBSA only exists in $60 \%$ of the cases. The findings from the present study using CEUS examination supported the results of $\mathrm{Ma}$ et al that the SCA exhibited considerable anatomical variations and the TBSAs with good quality which could be used as pedicle existed in 18 of the $26(69.2 \%)$ flaps. Therefore, the preoperative examination of the vessels is essential for successful surgery.

An ideal perforator mapping modality should be noninvasive; predict the presence and location of the perforators with high accuracy; provide the static and dynamic characteristics of the perforators, such as its caliber, pedicle length, pivot point position and PSV; and deliver a $3 \mathrm{D}$ image showing the course of the vessels and their surrounding anatomical relationships. The HHD, CDUS, CTA, and MRA are the most commonly used methods in preoperative perforator mapping nowadays. ${ }^{6,19,20}$ However, all of them are characterized by advantages and limitations. The HHD is a noninvasive and simple method. However, it can only detect vessels to a depth of $20 \mathrm{~mm}$ and does not give any information regarding the diameter or course of the vessels and its surrounding anatomy. The CDUS is noninvasive, economical, and can provide valuable hemodynamic and static information of the vessels. However, it does not provide a 3D image and lacks the ability to store image. Besides, although some studies have proven its high accuracy in identifying the perforators approximately $1 \mathrm{~mm}$ in diameter $\mathrm{r}^{7,19,21}$ for the identification of smaller perforators which are also valuable in pedicled flaps, CDUS always shows dotted, noncontinual signals and may cause high test-retest unreliability and high false-positive and false-negative rates. ${ }^{20}$ Multidetector CTA has been proposed as the best 

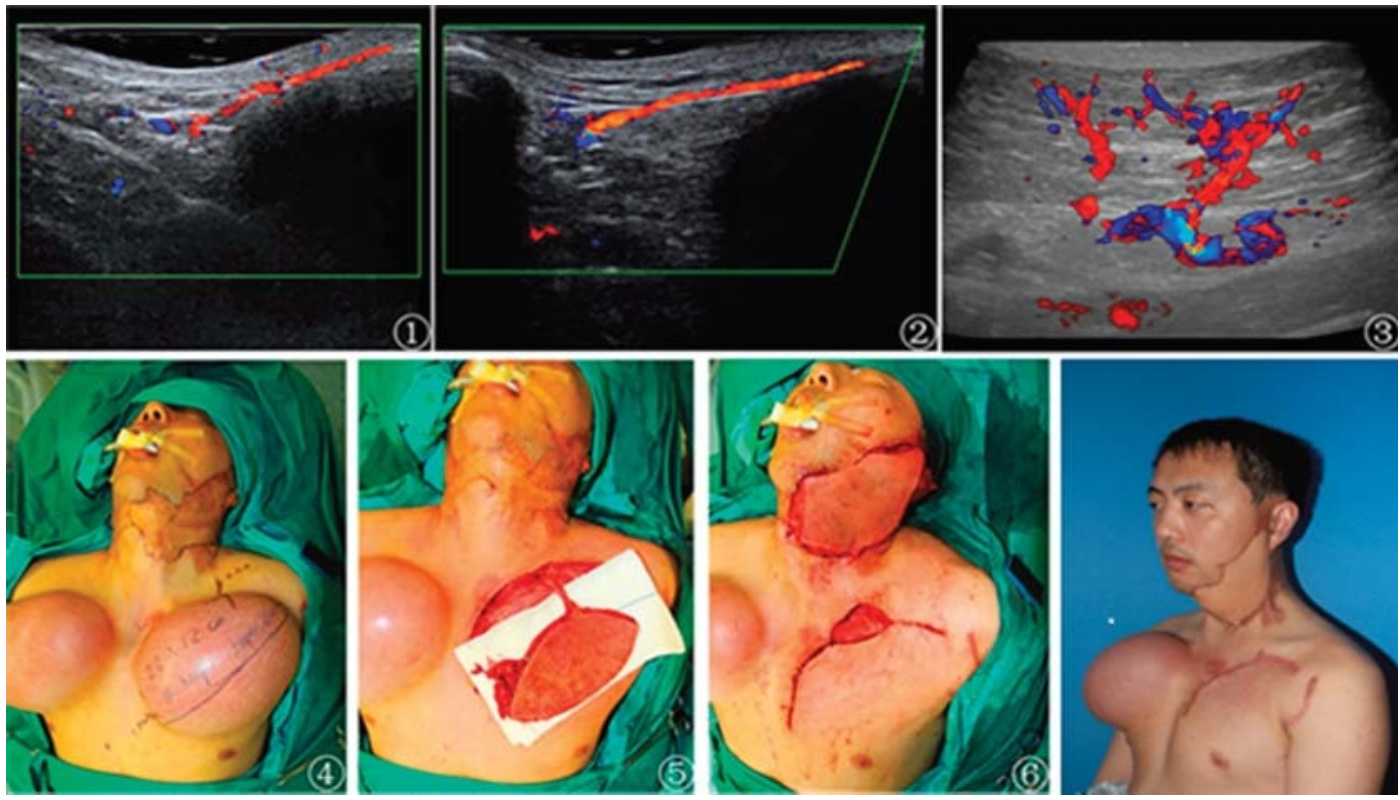

FIGURE 2. Case report. A 29-year-old man presented with postburn scar contractures of neck and lower face. Bilateral preexpanded pedicled TBSA flaps were planned to resurface the defect of neck and lower face. Left side was performed in the first stage. (1) CDUS examination revealed one left TBSA running in the subdermal layer of the expanded flap. (2) After injection of the contrast agent SonoVue, the CDFI signal of the TBSA increased. CEUS showed the TBSA more clearly. (3) With the 3D reconstruction method, we were able to visualize the all course of the TBSA and its root vessel, the left SC. (4) The location and course of the left TBSA was marked in the body surface. (5) During flap harvesting, the location of the TBSA was confirmed as identified preoperatively. (6) The flap was successfully transferred, while the donor site was closed primarily. (7) The flap survived completely after 2 weeks.

method for evaluating the vessels before surgery ${ }^{22}$ because it can provide an overall 3D image record showing the complete course of the vessels associated with their branch relationship and distribution in soft tissue. Besides, according to the technical development (256- or even 320-row CT), it has the capacity to identify the very small perforators with high accuracy. ${ }^{23,24}$ However, the primary disadvantage of CTA is the inevitable exposure to ionizing radiation and its subsequent risk of inducing complications. ${ }^{9,10}$ Also, it cannot provide any hemodynamic information, which is important parameter in preoperative planning. Although MRA can provide a 3D image of the vessels and has no radiation exposure, it is very expensive and will only detect vessels larger than $1.0 \mathrm{~mm}$, whereas TBSA are sometimes less than $1.0 \mathrm{~mm}{ }^{12}$ Therefore, new methods for preoperative perforator assessment should be explored.

Contrast-enhanced ultrasound was first used in detecting perforators in 2010 by Lu et al. ${ }^{13}$ The contrast agent SonoVue based on sulfur hexafluoride-microbubbles raises the blood echo and enhances the signal-to-noise ratio to improve the vessel image. ${ }^{14} \mathrm{Lu}$ et al ${ }^{13}$ demonstrated the effectiveness of CEUS because the perforators were displayed more clearly by CEUS than CDUS. However, that article was structured as a report of a series of cases, without evaluating these 2 methods scientifically. Later, $\mathrm{Su}$ et $\mathrm{al}^{25}$ performed a retrospective study including 32 patients undergoing different types of perforator flap reconstruction to analyze the value of CEUS combined with $3 \mathrm{D}$ reconstruction in preoperative perforator mapping and concluded that it was a valuable method with high specificity $(100 \%)$ and sensitivity. In the present study, we support these results and demonstrate the $100 \%$ accuracy of CEUS in detecting the TBSAs because all the TBSAs found by CEUS were confirmed during operation with no more TBSAs found intraoperatively, whereas the traditional CDUS had lower sensitivity and negative predictive value than that of CEUS. According to our investigation, the CEUS could detect the perforator as small as $0.5 \mathrm{~mm}$, whereas the CDUS showed dotted signal even for the perforators of
0.5 to $0.6 \mathrm{~mm}$ and could not detect smaller vessels. Although our present study demonstrates $100 \%$ specificity for both CDUS and CEUS with $3 \mathrm{D}$ reconstruction in detecting TBSAs for detecting the perforators in the sites with more complicated anatomical structures, the CEUS may have higher specificity because the continuous 3D image could be seen which help the surgeons better view all course of the vessel and predict the root vessel. This could also help the surgeons decrease surgery time and successfully elevated the flaps without concern for injuring the vessels.

Besides the advantages of higher accuracy of CEUS over CDUS in detecting small perforators and providing 3D image of the vessels, CEUS has advantages over CTA or MRA by providing hemodynamic information. Peak systolic velocity is one of the most important perforator quality indicators. It is the maximum velocity of the blood flow at the end of the systolic period. We did not find statistically significant difference between CDUS and CEUS for the assessment of PSV in this present study and had recognized the correlation between the PSV and flap morbidity in our previous work. According to our experience, the TBSA with PSV above $10 \mathrm{~cm} / \mathrm{sec}$ could be selected as the pedicle of TBSA flap. ${ }^{2}$ Therefore, although we found the existence of TBSAs in 20 flaps, for 2 of the 20 flaps in which the PSV of TBSAs were less than $10 \mathrm{~cm} / \mathrm{s}$, IMAP flaps were indicated to prevent flap morbidity.

In addition, CEUS also has the advantages of being economical, minimally invasive, and avoiding exposure of the patient to radiation. In our present study of 20 patients, complications related to the contrast agent included minor gastrointestinal uncomfortable in 1 patient who recovered within 1 day without special treatment, which preliminarily confirmed the safety of CEUS.

The CEUS with 3D reconstruction still has its disadvantages. In our present study, we found statistically significant differences between CDUS and CEUS for the assessment of the caliber of the TBSAs. The CDUS has been considered reliable for caliber estimation by some technologists, ${ }^{19,26}$ whereas the results of CEUS seemed to be less reliable, 
which may be due to its CDFI signal excess. ${ }^{25}$ In 1 case of our study, the TBSA detected by CEUS with a caliber of $0.6 \mathrm{~mm}$ were found to be too thin during surgery, and we had to change our operative planning to elevate a free IMAP flap. In addition, CEUS is an operator dependent and time-consuming procedure, because it might take up to 1 hour for detecting TBSA, which is 30 to 45 minutes longer than CDUS. Furthermore, the small size of the volume probe limits the resulting image in width and depth, thus rendering it less accurate in detecting the deep vascular system. ${ }^{25}$

\section{ACKNOWLEDGMENT}

Informed consent was received for publication of the figures in this article.

\section{REFERENCES}

1. Li Q, Zan T, Gu B, et al. Face resurfacing using a cervicothoracic skin flap prefabricated by lateral thigh fascial flap and tissue expander. Microsurgery. 2009;29:515-523.

2. Zan T, Li H, Du Z, et al. Reconstruction of the face and neck with different types of pre-expanded anterior chest flaps: a comprehensive strategy for multiple techniques. J Plast Reconstr Aesthet Surg. 2013;66:1074-1081.

3. Cordova A, Pirrello R, D'Arpa S, et al. Vascular anatomy of the supraclavicular area revisited: feasibility of the free supraclavicular perforator flap. Plast Reconstr Surg. 2008;122:1399-1409.

4. Xie F, Wang J, Li Q, et al. Resurfacing large skin defects of the face and neck with expanded subclavicular flaps pedicled by the thoracic branch of the supraclavicular artery. Burns. 2012;38:924-930.

5. Ma X, Zheng Y, Xia W, et al. An anatomical study with clinical application of one branch of the supraclavicular artery. Clin Anat. 2009;22:215-220.

6. Smit JM, Klein S, Werker PM. An overview of methods for vascular mapping in the planning of free flaps. J Plast Reconstr Aesthet Surg. 2010;63:e674-e682.

7. Dorfman D, Pu LL. The value of color duplex imaging for planning and performing a free anterolateral thigh perforator flap. Ann Plast Surg. 2014;72 (suppl 1):S6-S8.

8. Schaverien MV, Ludman CN, Neil-Dwyer J, et al. Contrast-enhanced magnetic resonance angiography for preoperative imaging of deep inferior epigastric artery perforator flaps: advantages and disadvantages compared with computed tomography angiography: a United Kingdom perspective. Ann Plast Surg. 2011;67: 671-674.

9. Rozen WM, Whitaker IS, Stella DL, et al. The radiation exposure of computed tomographic angiography (CTA) in DIEP flap planning: low dose but high impact. J Plast Reconstr Aesthet Surg. 2009;62:e654-e655.
10. ten Dam MA, Wetzels JF. Toxicity of contrast media: an update. Neth J Med. 2008;66:416-422.

11. Rozen WM, Pan WR, Le Roux CM, et al. The venous anatomy of the anterior abdominal wall: an anatomical and clinical study. Plast Reconstr Surg. 2009;124: 848-853.

12. Rozen WM, Ashton MW, Stella DL, et al. Magnetic resonance angiography and computed tomographic angiography for free fibular flap transfer. $J$ Reconst Microsurg. 2008;24:457-458.

13. Lu LG, Xu ZZ, Liu JB, et al. A pilot study of contrast-enhanced ultrasound on the vessel perforators of skin flap. Shanghai Med Imag. 2010;19:3.

14. Schneider M, Arditi M, Barrau MB, et al. BR1: a new ultrasonographic contrast agent based on sulfur hexafluoride filled microbubbles. Invest Radiol. 1995;30: 451-457.

15. Palmer JH, Taylor GI. The vascular territories of the anterior chest wall. Br J Plast Surg. 1986;39:287-299.

16. Zhang YX, Yongjie H, Messmer C, et al. Thoracoacromial artery perforator flap: anatomical basis and clinical applications. Plast Reconstr Surg. 2013;131: $759 \mathrm{e}-770 \mathrm{e}$

17. Ma XJ, Lu KH, Ai YF. Anatomical studies on the design of a new cervicothoracic flap pedicled with transverse cervical artery. Chin J Clin Anat. 1994;12:2.

18. Pallua N, Wolter TP. Moving forwards: the anterior supraclavicular artery perforator (a-SAP) flap: A new pedicled or free perforator flap based on the anterior supraclavicular vessels. J Plast Reconstr Aesthet Surg. 2013;66:489-496.

19. Cina A, Salgarello M, Barone-Adesi L, et al. Planning breast reconstruction with deep inferior epigastric artery perforating vessels: multidetector CT angiography versus color Doppler US. Radiology. 2010;255:979-987.

20. Rozen WM, Phillips TJ, Ashton MW, et al. Preoperative imaging for DIEA perforator flaps: a comparative study of computed tomographic angiography and Doppler ultrasound. Plast Reconstr Surg. 2008;121:9-16.

21. Tsukino A, Kurachi K, Inamiya T, et al. Preoperative color Doppler assessment in planning of anterolateral thigh flaps. Plast Reconstr Surg. 2004;113:241-246.

22. Smit JM, Dimopoulou A, Liss AG, et al. Preoperative CT angiography reduce surgery time in perforator flap reconstruction. J Plast Reconstr Aesthet Surg. 2009;62:1112-1117.

23. Ono S, Chung KC, Hayashi H, et al. Application of multidetector-row computed tomography in propeller flap planning. Plast Reconstr Surg. 2011;127(2): 703-711.

24. Rozen WM, Ashton MW, Stella DL, et al. The accuracy of computed tomographic angiography for mapping the perforators of the deep inferior epigastric artery: a blinded, prospective cohort study. Plast Reconstr Surg. 2008;122:1003-1009.

25. Su W, Lu L, Lazzeri D, et al. Contrast-enhanced ultrasound combined with threedimensional reconstruction in preoperative perforator flap planning. Plast Reconstr Surg. 2013;131:80-93.

26. Stupp T, Pavlidis M, Busse $H$, et al. Presurgical and postsurgical ultrasound assessment of lacrimal drainage dysfunction. Am J Ophthalmol. 2004;138:764-771. 